\title{
碱式氯化镁晶须制备纳米氧化镁热分解动力学研究
}

\author{
苟生莲 ${ }^{1,2,3}$, 乃学瑛 ${ }^{1}$, 肖剑飞 ${ }^{1}$, 叶俊伟 ${ }^{4}$, 董亚萍 ${ }^{1}$, 李 武 ${ }^{1,2}$
}

(1. 中国科学院 青海盐湖研究所 中国科学院盐湖资源综合高效利用重点实验室, 西宁 $810008 ; 2$. 青海省盐湖资 源化学重点实验室, 西宁 $810008 ; 3$. 中国科学院大学, 北京 $100049 ; 4$. 大连理工大学 化工学院, 大连 116024)

摘 要: 采用水热法以氯化镁和氢氧化钲为原料制备了碱式氯化镁(BMC)晶须, 然后热解得到了纳米氧化镁。经透 射电镜(TEM)和选区电子衍射(SAED)分析其粒径在 $20 \sim 40 \mathrm{~nm}$ 之间, 暴露晶面族为 $\{111\}$ 和 $\{110\}$ 。通过热重差热分 析(TG-DTA)、扫描电镜(SEM)、X 射线衍射(XRD)以及红外光谱(FT-IR)分析确定了碱式氯化镁晶须热分解过程分 四步进行, 前两步分别脱去两个结晶水, 第三步脱氯化氢, 最后脱羟基水。采用 Satava 法和微分法对 BMC 晶须的 热分解机理和动力学进行了研究, 得出第一步反应热分解机理为随机成核与随后生长、第二步为二维扩散、第三步 为相边界反应、第四步为一维相边界反应。

关 键 词: 碱式氯化镁; 纳米氧化镁; 热分解; 晶须; 水热法

中图分类号: TQ132 文献标识码: A

\section{Preparation and Thermal Decomposition of Basic Magnesium Chloride Whiskers}

\author{
GOU Sheng-Lian ${ }^{1,2,3}$, NAI Xue-Ying ${ }^{1}$, XIAO Jian-Fei $^{1}$, YE Jun-Wei ${ }^{4}$, DONG Ya-Ping ${ }^{1}$, LI Wu ${ }^{1,2}$
}

(1. Key Laboratory of Comprehensive and Highly Efficient Utilization of Salt Lake Resources, Qinghai Institute of Salt Lakes, Chinese Academy of Sciences, Xining 810008, China; 2. Key Laboratory of Salt Lake Resources Chemistry of Qinghai Province, Xining 810008, China; 3. University of Chinese Academy of Sciences, Beijing 100049, China; 4. School of Chemical Engineering, Dalian University of Technology, Dalian 116024, China)

\begin{abstract}
Basic magnesium chloride (BMC) whiskers were prepared by hydrothermal method using magnesium chloride and calcium hydroxide as raw materials. Subsequently, nano-MgO was obtained by pyrolysis of the resulting whisker. Transmission electron microscopy (TEM) images showed that the diameter of nano-MgO was between 20 and $40 \mathrm{~nm}$. The corresponding selected-area electron diffraction (SAED) pattern demonstrated the singlecrystalline nature with the exposed plane of $\{111\}$ and $\{110\}$. TG-DTG, SEM, XRD, and FT-IR analysis confirmed the four-step pyrolysis processes of BMC whisker. Results showed two molecules of crystal water lost during the first and second steps, respectively. Subsequently, two hydrogen chloride molecules were released in the third step. Finally, hydroxy water was dehydrated. The thermal decomposition kinetics of BMC whisker was studied by means of Satava and differential method. It indicates that the first step of the thermal decomposition of BMC whiskers is random nucleation and subsequent growth mechanism. The second step is two-dimensional diffusion. The third and the final steps are phase boundary reaction and one-dimensional phase boundary reaction mechanism, respectively.
\end{abstract}

Key words: basic magnesium chloride; nano magnesium oxide; thermal decomposition; whiskers; hydrothermal method

收稿日期：2018-10-26; 收到修改稿日期：2019-01-08

基金项目: 国家自然科学基金柴达木盐湖化工科学研究联合基金(U1607101); 青海省科技厅项目(2019-ZJ-7029)

NSFC-Qaidam Salt Lake Chemical Science Research Joint Fund (U1607101); Qinghai Provincial Science and Technology Department Project (2019-ZJ-7029)

作者简介: 苟生莲(1992-), 女，硕士研究生. E-mail: goushenglian@163.com

通讯作者: 李 武, 研究员. E-mail: driverlaoli@163.com 
纳米 $\mathrm{MgO}$ 作为镁资源的一种重要利用形式, 由于粒径小、比表面积大具有不同于本体材料的光、 电、热以及力学性能, 可应用于催化、陶瓷、耐火 材料、吸附材料、补强剂以及抗菌材料等邻域 ${ }^{[1-2]}$ 。 目前, 制备纳米氧化镁的方法主要包括固相法 ${ }^{[3]} 、$ 沉 淀法 $^{[4]}$ 、前驱体法 ${ }^{[5-6]}$ 、水热法 ${ }^{[7-8]}$ 和溶胶-凝胶法 ${ }^{[9-10]}$ 等。其中, 前驱体法因具有产品收率高、过程易控 制等优点而引起广泛关注。碱式氯化镁作为制备纳 米氧化镁的前驱体之一, 具有易制备、产率高、分 解温度低、产物粒径均匀及分散性好等特点, 其结 构通式为 $x \mathrm{Mg}(\mathrm{OH})_{2} \cdot y \mathrm{MgCl}_{2} \cdot z \mathrm{H}_{2} \mathrm{O}$, 可简写为 $x y z$ 型 $\mathrm{BMC}$, 根据制备条件的不同可以得到 $2 \mathrm{Mg}(\mathrm{OH})_{2} \cdot \mathrm{MgCl}_{2} \cdot 2 \mathrm{H}_{2} \mathrm{O} 、 3 \mathrm{Mg}(\mathrm{OH})_{2} \cdot \mathrm{MgCl}_{2} \cdot 8 \mathrm{H}_{2} \mathrm{O}$ 、 $5 \mathrm{Mg}(\mathrm{OH})_{2} \cdot \mathrm{MgCl}_{2} \cdot 8 \mathrm{H}_{2} \mathrm{O} 、 9 \mathrm{Mg}(\mathrm{OH})_{2} \cdot \mathrm{MgCl}_{2} \cdot 5 \mathrm{H}_{2} \mathrm{O}$ 以 及 $9 \mathrm{Mg}(\mathrm{OH})_{2} \cdot \mathrm{MgCl}_{2} \cdot 4 \mathrm{H}_{2} \mathrm{O}$ 相 ${ }^{[11-13]}$ 。

用碱式氯化镁制备纳米氧化镁时，因前驱体的 相变温度与其晶体尺寸和结构相关, 并且氧化镁的 形貌、比表面积、热稳定性和化学稳定性与前驱体 的热解条件也密切相关, 所以, 以 $\mathrm{BMC}$ 晶须为前驱 体制备纳米氧化镁, 需对 BMC 的热解过程和热解 动力学进行研究, 以便通过控制 BMC 的热解条件 得到目标纳米氧化镁。

本实验采用水热法制备出 914 型碱式氯化镁 $\left(9 \mathrm{Mg}(\mathrm{OH})_{2} \cdot \mathrm{MgCl}_{2} \cdot 4 \mathrm{H}_{2} \mathrm{O}\right)$, 热解得到了纳米级氧化 镁。对 $\mathrm{BMC}$ 的热分解过程、热解机理及热解动力 学进行了分析研究。

\section{1 实验方法}

\section{1 制备前驱体 BMC}

配置 $40 \mathrm{~mL}$ 浓度为 $4 \mathrm{~mol} / \mathrm{L}$ 的氯化镁溶液, 加 入 $10 \mathrm{~mL}$ 固液比为 $1: 10$ 的氢氧化钻料浆, 搅拌均 匀后将料浆转移至 $100 \mathrm{~mL}$ 反应釜中, 将其置于均 相反应器内, 在 $160{ }^{\circ} \mathrm{C}$ 水热反应 $2 \mathrm{~h}$ 。然后自然冷却 至室温, 经抽滤、洗涤和干燥得到絮状产物。经 XRD 及 SEM 分析(如图 1), 产物为直径在 $100 \mathrm{~nm}$ 左右, 长度 50 70 $\mu \mathrm{m}$ 的长纤维, 结晶度良好且衍射峰与 $9 \mathrm{Mg}(\mathrm{OH})_{2} \cdot \mathrm{MgCl}_{2} \cdot 4 \mathrm{H}_{2} \mathrm{O}(914$ 型 $\mathrm{BMC})$ 的标准卡相 吻合。

\section{2 前驱体热解实验}

将上述 $\mathrm{BMC}$ 产物放置于马弗炉中, 在空气氛 围中以 $10{ }^{\circ} \mathrm{C} / \mathrm{min}$ 的升温速度分别升温至 $220 、 300$ 、 340 和 $500{ }^{\circ} \mathrm{C}$, 不经过恒温阶段直接取出灼烧产物, 对产物进行 SEM、XRD、TEM 以及 FT-IR 分析。
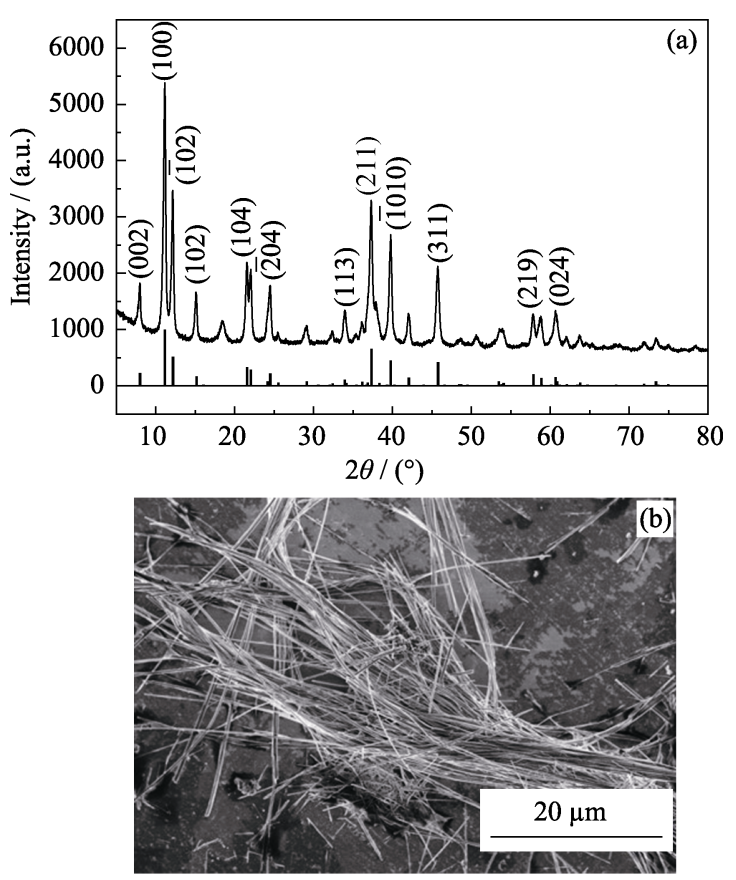

图 1 样品的 XRD 图谱(a)和 SEM 照片(b)

Fig. 1 XRD patten (a) and SEM image (b) of the sample

\section{2 结果与讨论}

\section{1 热分解过程分析}

\subsubsection{TG-DTA}

对前驱体 BMC 进行 TG-DTA(如图 2)分析可知, $\mathrm{BMC}$ 的热分解过程分四步进行, 温度范围分别为 $60.8 \sim 220{ }^{\circ} \mathrm{C} 、 220 \sim 300{ }^{\circ} \mathrm{C} 、 300 \sim 340{ }^{\circ} \mathrm{C}$ 和 $340 \sim 820{ }^{\circ} \mathrm{C}$, 峰值温度分别为 $78.0 、 251.3 、 313.4$ 和 $382.5{ }^{\circ} \mathrm{C}$, 失 重率分别为 $5.28 \% 、 6.56 \% 、 9.43 \%$ 和 $22.92 \%$ 。初步 推测前两个阶段分别失去两个水分子, 理论失重率 都为 $5.22 \%$; 第三阶段 BMC 分子中的氯化镁与羟基 水反应，分子中脱去氯化氢，理论失重率为 $10.59 \%$; 第四阶段分子中脱去剩余的羟基水, 生成氧化镁, 此阶段理论失重率为 $20.90 \%$ 。

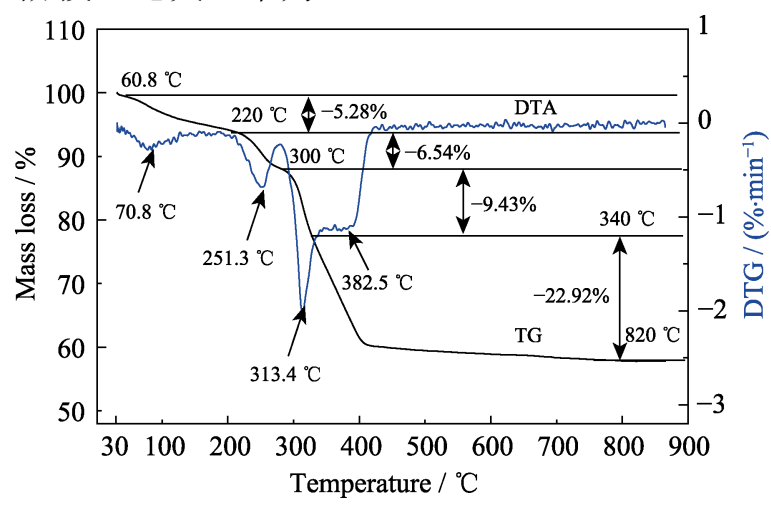

图 2 样品的 TG-DTA 分析结果

Fig. 2 TG-DTA curves of the sample 


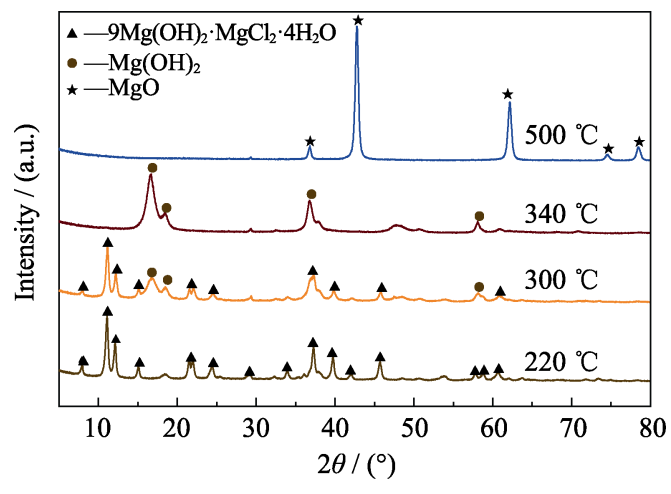

图 3 样品经不同温度灼烧后产物的 XRD 图谱

Fig. 3 XRD patterns of the products burned at different temperatures

\subsection{2 热解产物形貌及晶型}

根据 TG-DTG 分析, BMC 热解各阶段实际失重 率与理论失重率有一定的误差, 需进一步验证结 果。因此对前驱体 BMC 进行 220、300、340 和 $500{ }^{\circ} \mathrm{C}$ 的灼烧实验，分析产物形貌与晶型的变化规律。

由图 3 和图 4 可知，前驱体经 220 和 $300{ }^{\circ} \mathrm{C}$ 灼烧 后，形貌依然保持纤维状和 BMC 的晶体结构，说明 前两阶段从前驱体中脱去结晶水生成 $9 \mathrm{Mg}(\mathrm{OH})_{2} \cdot \mathrm{MgCl}_{2}$, 维持 $\mathrm{BMC}$ 的基本晶格骨架而未收缩坞塌; 当温度 升高至 $340{ }^{\circ} \mathrm{C}$ 时, 样品的晶体结构转化为氢氧化镁 晶型，前驱体的纤维状形貌断裂，出现短棒状产物， 且直径减小，说明该阶段 BMC 中氯化镁与羟基反 应脱去氯化氢，生成氢氧化镁及氧化镁混合物。其 中氧化镁应属于无定型状态未出现于 XRD 图谱中, 而 $\mathrm{BMC}$ 分子中美氧多面体成核、结晶形成氢氧化 镁晶体; 最后, 前驱体经 $500{ }^{\circ} \mathrm{C}$ 灼烧后转化为氧化 镁晶型, 产物由纤维状断裂成颗粒状, 说明氢氧化 镁热解脱去羟基水, 前驱体晶格收缩坞塌幅度较大, 难以维持基本骨架, 所得氧化镁粒径较小。最终产 物经 TEM 及电子衍射分析可知(如图 5), 产物氧化 镁直径在 20 40 $\mathrm{nm}$ 之间, 由于 BMC 热解第三阶段脱
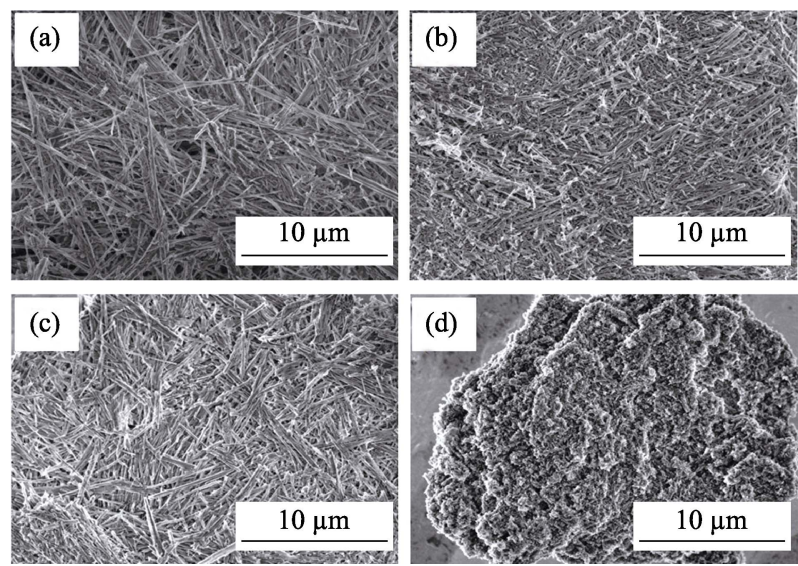

图 4 样品经不同温度灼烧后产物的 SEM 照片

Fig. 4 SEM images of the products burned at different temperatures (a) $220{ }^{\circ} \mathrm{C}$; (b) $300{ }^{\circ} \mathrm{C}$; (c) $340{ }^{\circ} \mathrm{C}$; (d) $500{ }^{\circ} \mathrm{C}$
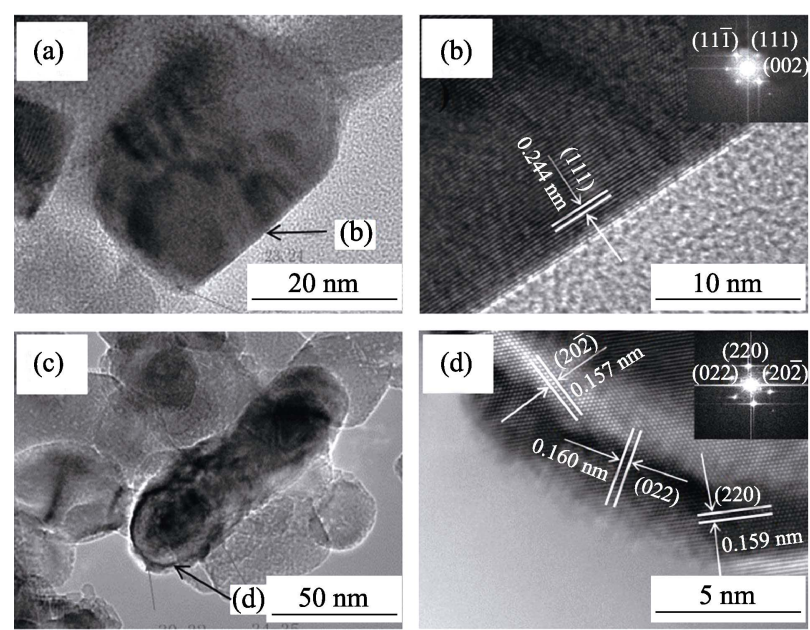

图 5 经 $500{ }^{\circ} \mathrm{C}$ 灼烧后产物的 $\mathrm{TEM}$ 照片及电子衍射分析结果

Fig. 5 TEM images and SAED patterns of the product burned at $500{ }^{\circ} \mathrm{C}$

去氯化氢时形成的氧化镁与第四阶段脱羟基水时生成 的氧化镁暴露的晶面族不同，所得到的氧化镁暴露晶 面族有两种，分别为 $\{111\}$ (图 5(b)) 和 $\{110\}$ (图 5(d))。

\subsection{3 官能团变化}

BMC 各阶段分解产物主要是脱去不同类型的 水分子与氯化氢, 通过红外分析可研究热解各阶段 产物官能团变化规律。从图 6(a)中可以看出, 在 $3695 \mathrm{~cm}^{-1}$ 处有个小峰, 这是 $\mathrm{BMC}$ 中 $\mathrm{H}-\mathrm{O} \cdot \mathrm{Mg}$ 的 伸缩振动峰, 而在 $3630 \mathrm{~cm}^{-1}$ 处的峰为 $\mathrm{H}-\mathrm{O} \cdot \cdot \mathrm{Mg}$ 的 缔合峰 ${ }^{[14]}$; 在 $3551 \mathrm{~cm}^{-1}$ 处的峰为 $\mathrm{H}-\mathrm{O} \cdot \cdot \mathrm{Cl}$ 的伸缩 振动峰 ${ }^{[15]}$; 在 3439 和 $1633 \mathrm{~cm}^{-1}$ 处的峰分别为吸附 水中 $\mathrm{O}-\mathrm{H}$ 的伸缩振动峰和 $\mathrm{H}-\mathrm{O}-\mathrm{H}$ 的弯曲振动峰 ${ }^{[16]}$; 在 $1439 \mathrm{~cm}^{-1}$ 处的峰归属于 $\mathrm{CO}_{3}{ }^{2-}$ 的吸收峰，该峰的 出现可能是由于原料中的杂质, 或者 $\mathrm{Mg}(\mathrm{OH})_{2} / \mathrm{MgO}$ 与 吸附在样品表面的 $\mathrm{H}_{2} \mathrm{O}$ 和 $\mathrm{CO}_{2}$ 反应生成了碱式碳酸 镁/碳酸镁, 661 和 $595 \mathrm{~cm}^{-1}$ 处的峰分别归属于结晶 水的 $\omega_{\mathrm{H}-\mathrm{O}-\mathrm{H}}$ 和 $\gamma_{\mathrm{H}-\mathrm{O}-\mathrm{H}}$; 而在 $506 \mathrm{~cm}^{-1}$ 左右的峰归属 于 $\mathrm{BMC}$ 中 $v_{\mathrm{Mg}-\mathrm{O}}{ }^{[17]}$ 。

由图 6 可以看出, 在 661 和 $595 \mathrm{~cm}^{-1}$ 处的 $\omega_{\mathrm{H}-\mathrm{O}-\mathrm{H}}$

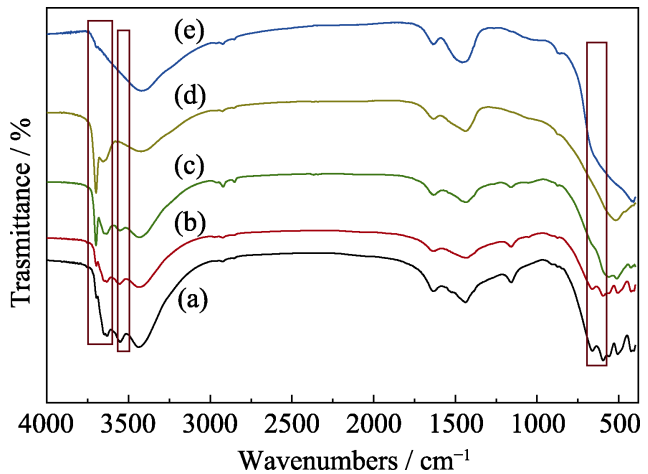

图 6 经不同温度灼烧后产物的 FT-IR 谱图

Fig. 6 FT-IR spectra of the products burned at different temperatures

(a) BMC; (b) $220{ }^{\circ} \mathrm{C}$; (c) $300{ }^{\circ} \mathrm{C}$; (d) $340{ }^{\circ} \mathrm{C}$; (e) $500{ }^{\circ} \mathrm{C}$ 
和 $\gamma_{\mathrm{H}-\mathrm{O}-\mathrm{H}}$ 在 (c e) 中消失, 说明升温至 $300{ }^{\circ} \mathrm{C}$ 时样品 中的结晶水已全部失去; 在 $3551 \mathrm{~cm}^{-1}$ 处的 $v_{\mathrm{H}-\mathrm{O} \cdot{ }^{\circ} \mathrm{Cl}}$ 在 (d e)中消失, 说明升温至 $340{ }^{\circ} \mathrm{C}$ 时样品中的 $\mathrm{Cl}$ 已 全部失去; 在 $3695 \mathrm{~cm}^{-1}$ 处的峰强度及波数随热解 程度的增加而增强, 而 $3630 \mathrm{~cm}^{-1}$ 处的峰随热解程 度的增加而减弱, 说明随着热解的进行, $\mathrm{BMC}$ 中小 分子 $\mathrm{H}_{2} \mathrm{O}$ 及 $\mathrm{HCl}$ 逐步从 $\mathrm{BMC}$ 晶格中脱离, 结合图 4 扫描电镜分析可以看出 $220 、 300 、 340{ }^{\circ} \mathrm{C}$ 的热解产 物基本维持晶须形貌, 产物在各阶段原位结晶且晶格 缺陷增多, 导致 $\mathrm{H}-\mathrm{O} \cdot \cdot \mathrm{Mg}$ 单体增多缔合减少, 氢键 作用减弱; 当热解至 $500{ }^{\circ} \mathrm{C}$ 时, 图 6(e)中 $3695 \mathrm{~cm}^{-1}$ 峰 几乎消失, 说明 $\mathrm{Mg}(\mathrm{OH})_{2}$ 失去羟基水转变成 $\mathrm{MgO}$, 同时 $419 \mathrm{~cm}^{-1}$ 处出现 $\mathrm{Mg}-\mathrm{O}$ 振动峰, 与本征态氧化 镁特征峰相比发生了红移 ${ }^{[18]}$ 。

因此, 通过以上实验分析得出 BMC 晶须的热 分解过程分为四步进行, 中间产物为 $\mathrm{Mg}(\mathrm{OH})_{2}$, 最 终产物是 $\mathrm{MgO}$, 反应方程式如下:

$$
\begin{gathered}
\text { 第一步: } 9 \mathrm{Mg}(\mathrm{OH})_{2} \cdot \mathrm{MgCl}_{2} \cdot 4 \mathrm{H}_{2} \mathrm{O} \rightarrow \\
9 \mathrm{Mg}(\mathrm{OH})_{2} \cdot \mathrm{MgCl}_{2} \cdot 2 \mathrm{H}_{2} \mathrm{O}+2 \mathrm{H}_{2} \mathrm{O} \uparrow \\
\text { 第二步: } 9 \mathrm{Mg}(\mathrm{OH})_{2} \cdot \mathrm{MgCl}_{2} \cdot 2 \mathrm{H}_{2} \mathrm{O} \rightarrow \\
9 \mathrm{Mg}(\mathrm{OH})_{2} \cdot \mathrm{MgCl}_{2}+2 \mathrm{H}_{2} \mathrm{O} \uparrow \\
\text { 第三步: } 9 \mathrm{Mg}(\mathrm{OH})_{2} \cdot \mathrm{MgCl}_{2} \rightarrow \\
8 \mathrm{Mg}(\mathrm{OH})_{2} \cdot 2 \mathrm{MgO}+2 \mathrm{HCl} \uparrow \\
\text { 第四步: } 8 \mathrm{Mg}(\mathrm{OH})_{2} \cdot 2 \mathrm{MgO} \rightarrow \\
10 \mathrm{MgO}+8 \mathrm{H}_{2} \mathrm{O} \uparrow
\end{gathered}
$$

\section{$2.2 \mathrm{BMC}$ 热分解动力学}

\subsection{1 热分解机理分析}

采用 Satava 法来确定 BMC 晶须的热分解机理, 假设 $\mathrm{BMC}$ 连续热解过程的每一步都存在一个对应 机理函数 $G(\alpha), G(\alpha)$ 对 $1 / T$ 作图得到的曲线越接近直 线, 拟合优度 $R^{2}$ 越接近于 1 , 则 $G(\alpha)$ 就是最概然机 理函数。式中 $\alpha=\left(m_{i}-m_{\tau}\right) / m, m_{i}$ 为样品的初始质量, $m_{\tau}$ 为温度 $T$ 时样品的质量, $m$ 为样品失重量。

对 30 种常用的动力学机理函数进行了拟合, 结 果如表 1 所示, 分析发现, 第一步热分解反应机理 函数 $\left\{G(\alpha)=[-\ln (1-\alpha)]^{3 / 4}\right\}$ 的拟合优度最高, $R^{2}$ 为 0.9991 , 推断热分解机理为随机成核与随后生长, 服从反应级数为 $3 / 4$ 的 Avrami-Erofeev 方程; 第二
步反应的最概然机理函数为 $\left\{G(\alpha)=\left[1-(1-\alpha)^{1 / 2}\right]^{1 / 2}\right\}$, $R^{2}$ 为 0.9978 , 推断热分解机理为二维扩散, 服从反 应级数为 $1 / 2$ 的 Jander 方程; 第三步反应的最概然 机理函数为 $\left[G(\alpha)=\alpha^{1 / 2}\right], R^{2}$ 为 0.9970 , 热分解机理为 相边界反应, 服从反应级数为 $1 / 2$ 的 Mampel Power 法则; 第四步反应机理函数 $[G(\alpha)=\alpha]$ 拟合优度最高, $R^{2}$ 为 0.9996 , 推断热分解反应机理为一维相边界反 应，服从反应级数为 1 的 Mampel Power 法则。

\subsection{2 动力学参数计算}

热分析动力学方法包括微分法和积分法两大类, 本研究采用微分法(Kissinger 法 $^{[19]}$ )来研究 BMC 晶 须的热分解动力学。根据 Kissinger 法则固体非等温 条件下热解的动力学方程式为:

$$
\frac{d \alpha}{\mathrm{d} T}=\frac{A}{\beta} \exp \left(\frac{E}{R T}\right) f(\alpha)
$$

式中 $\alpha$ 是分解程度, $t$ 是分解时间, $A$ 是指前因子, $\beta$ 是升温速率, $E$ 是活化能, $R$ 是摩尔气体常量, $n$ 是反 应级数, $\kappa$ 是速率常数, $f(\alpha)$ 为机理函数的微分式, 且 $f(\alpha)=1 / G^{\prime}(\alpha)$ 。根据 Kissinger 原理, 假设在峰值温度 下反应速度最快, 此时的温度命名为 $T_{\mathrm{p}}, \frac{E}{R T_{\mathrm{P}}^{2}}=$ $\frac{A}{\beta} n\left(1-\alpha_{\mathrm{P}}\right)^{(n-1)} \exp \left(-\frac{E}{R T_{\mathrm{P}}}\right)$, 式中 $n\left(1-\alpha_{\mathrm{P}}\right)^{n-1}$ 与 $\beta$ 无关, 且其值近似等于 1 , 因此将式改写为:

$$
\ln \left(\frac{\beta}{T_{\mathrm{P}}^{2}}\right)=\ln \frac{A R}{E}-\frac{E}{R T_{\mathrm{P}}}
$$

对于一个确定的反应, $A 、 R$ 和 $E$ 是定值, $\ln \left(\frac{\beta}{T_{\mathrm{P}}^{2}}\right)$ 对 $\frac{1}{T_{\mathrm{P}}}$ 作图得到一条直线, 可从直线的斜率求出 $E$, 从截距求出 $A$ 。形状指数 $S=\frac{a}{b}=0.63 n^{2}$, 式中 $a$ 和 $b$ 为 DTG 曲线中峰两侧的切线与顶部切线的交点到 中间轴的距离, 示意图如图 7 所示, 从而计算得到 $n$ 。

将实验数据带入上述公式计算得到表 2 。从表 2 中可以发现 $\mathrm{BMC}$ 纳米晶须热分解整体反应活化能 较低, 其中第一步反应活化能最低为 $38.16 \mathrm{~kJ} / \mathrm{mol}$,

表 $1 G(\alpha)$ 函数表达式和四步反应相关系数

Table 1 Algebraic expressions of $G(\alpha)$ and the correlation coefficients for the four decomposition steps

\begin{tabular}{ccccccc}
\hline \multirow{2}{*}{ Function name } & \multirow{2}{*}{ Mechanism } & $G(\alpha)$ & \multicolumn{4}{c}{$R^{2}$} \\
\cline { 3 - 8 } & & & I & II & III & IV \\
\hline Avrami-Erofeev equation & Random nucleation and its subsequent growth & {$[-\ln (1-\alpha)]^{3 / 4}$} & 0.9991 & 0.9521 & 0.9129 & 0.9829 \\
Jander equation & Two-dimensional diffusion & {$\left[1-(1-\alpha)^{1 / 2}\right]^{1 / 2}$} & 0.9716 & 0.9978 & 0.9919 & 0.9957 \\
Mampel power low & Phase boundary reaction & $\alpha^{1 / 2}$ & 0.9598 & 0.9929 & 0.9970 & 0.9817 \\
Mampel power low & Phase boundary reaction(One-dimensional) & $\alpha$ & 0.9988 & 0.9932 & 0.9916 & 0.9996 \\
\hline
\end{tabular}




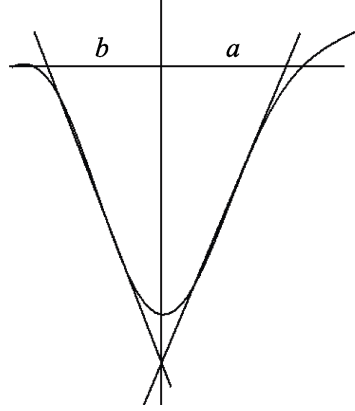

图 $7 a$ 和 $b$ 的关系图

Fig. 7 Diagram of $a$ and $b$

表明第一步反应最容易进行，可在较低温度下短时 间内脱去两个结晶水; 第二步反应活化能为 $152.35 \mathrm{~kJ} / \mathrm{mol}$ ，稍高于第一步反应; 第三步和第四 步反应活化能分别为 125.38 和 $85.52 \mathrm{~kJ} / \mathrm{mol}$ 。因此 判断 914 型碱式氯化镁纳米晶须热分解过程中第一 步是快反应，第二、三和四步反应是慢反应。

结合前面得出的最概然机理函数得出 BMC 热 分解过程中四步反应的动力学方程为:

$$
\begin{aligned}
& \text { 第一步: } \frac{\mathrm{d} \alpha}{\mathrm{d} T}=\frac{1.01 \times 10^{5}}{\beta} \cdot \mathrm{e}^{-\frac{4589.84}{T}} \cdot(1-\alpha)^{3 / 4} \\
& \text { 第二步: } \frac{\mathrm{d} \alpha}{\mathrm{d} T}=\frac{4.87 \times 10^{14}}{\beta} \cdot \mathrm{e}^{-\frac{18324.51}{T}} \cdot(1-\alpha)^{1 / 2} \\
& \text { 第三步: } \frac{\mathrm{d} \alpha}{\mathrm{d} T}=\frac{3.65 \times 10^{10}}{\beta} \cdot \mathrm{e}^{-\frac{15080.58}{T}} \cdot(1-\alpha)^{1 / 2} \\
& \text { 第四步: } \frac{\mathrm{d} \alpha}{\mathrm{d} T}=\frac{1.90 \times 10^{6}}{\beta} \cdot \mathrm{e}^{-\frac{10286.26}{T}} \cdot(1-\alpha)
\end{aligned}
$$

\section{3 结论}

采用水热法制备的碱式氯化镁晶须为 $9 \mathrm{Mg}(\mathrm{OH})_{2} \cdot \mathrm{MgCl}_{2} \cdot 4 \mathrm{H}_{2} \mathrm{O}$ 型, 其直径在 $100 \mathrm{~nm}$ 左右, 长度在 $50 \sim 70 \mu \mathrm{m}$, 将产物热解后得到了粒径在 20 40 nm 的纳米氧化镁。经计算分析得出 BMC 热 分解过程分四步进行, 第一步脱去两个水分子的反 应机理为随机成核和随后生长, 动力学方程为 $\frac{\mathrm{d} \alpha}{\mathrm{d} T}=\frac{1.01 \times 10^{5}}{\beta} \cdot \mathrm{e}^{-\frac{4589.84}{T}} \cdot(1-\alpha)^{3 / 4}$; 第二步也是脱去 两个水分子, 反应机理为二维扩散, 动力学方程为

表 2 BMC 晶须热分解动力学计算结果

Table 2 Dynamic calculation results of the thermal decomposition of BMC whiskers

\begin{tabular}{ccccc}
\hline Parameter & I & II & III & IV \\
\hline$n$ & 0.6 & 0.6 & 0.5 & 1.1 \\
$E /\left(\mathrm{kJ} \cdot \mathrm{mol}^{-1}\right)$ & 38.16 & 152.35 & 125.38 & 85.52 \\
$A / \mathrm{min}^{-1}$ & $1.01 \times 10^{5}$ & $4.87 \times 10^{14}$ & $3.65 \times 10^{10}$ & $1.90 \times 10^{6}$ \\
\hline
\end{tabular}

$\frac{\mathrm{d} \alpha}{\mathrm{d} T}=\frac{4.87 \times 10^{14}}{\beta} \cdot \mathrm{e}^{-\frac{18324.51}{T}} \cdot(1-\alpha)^{1 / 2}$; 第三步脱除两

个 $\mathrm{HCl}$, 反应机理为相边界反应, 动力学方程为 $\frac{\mathrm{d} \alpha}{\mathrm{d} T}=\frac{3.65 \times 10^{10}}{\beta} \cdot \mathrm{e}^{-\frac{15080.58}{T}} \cdot(1-\alpha)^{1 / 2}$; 第四步反应是 脱除八个羟基水得到最终产物 $\mathrm{MgO}$, 反应机理为一 维相边界反应, 动力学方程为 $\frac{\mathrm{d} \alpha}{\mathrm{d} T}=\frac{1.90 \times 10^{6}}{\beta}$. $\mathrm{e}^{-\frac{10286.26}{T}} \cdot(1-\alpha)$ 。

\section{参考文献}

[1] ZHANG W, WANG B H. Progress on preparation on nano-structured magnesia power. China Powder Science and Technology, 2004, 10(2): 40-44.

[2] 易师甜. 纳米氧化镁的制备及抗菌性能研究. 武汉: 华中科技 大学硕士学位论文, 2009.

[3] LIAO L L, LIU J P. Solid phase synthesis of nano-structured magnesia. Journal of Functional Materials, 2001, 18(12): 696-698.

[4] GAO Z J, QIN B J, FEN G L. Study on preparation of nanometer $\mathrm{MgO}$ by homogeneous precipitation method. PaperScience \& Technology, 2013, 32(4): 53-56.

[5] WANG X L, XUE D F. Direct observation of the shape evolution of $\mathrm{MgO}$ whiskers in a solution system. Materials Letters, 2006, 60(25): 3160-3164.

[6] LU S Y, XIN S Q, CAI R H, et al. Progress in precursor preparation method of magnesium oxide whisker. Journal of Salt and Chemical Industry, 2013, 4(42): 5-9.

[7] WANG J Y, ZHAO L Q, LIU Z H, et al. Mesoporous magnesium oxide of different morphology by hydrothermal method and their antibacterial performance. Chemical Research and Application, 2015, 27(10): 1477-1483.

[8] 王佩佩. 纳米氢氧化镁晶须的制备与生长机理研究. 呼和浩特: 内蒙古工业大学硕士学位论文, 2010.

[9] LIU Z. Preparation of nanometer MgO by Sol-Gel method from magnesite. Applied Chemical Industry, 2012, 41(5): 837-839.

[10] MASTULIA S, ANSARIA S, NAWAWIA A, et al. Effects of cationic surfactant in Sol-Gel synthesis of nano sized magnesium oxide. APCBEE Procedia, 2012, 3(8): 93-98.

[11] GOGOTSI G A. Criteria of ceramics fracture (edge chipping and fracture toughness tests). Ceramics International, 2013, 39(3): 3293-3300.

[12] CHRISTENSEN A N, NORBY P, HANSON J C. Chemical reactions in the system $\mathrm{MgO}-\mathrm{MgCl}_{2}-\mathrm{H}_{2} \mathrm{O}$ followed by time-resolved synchrotron $X$-ray powder diffraction. Journal of Solid State Chemistry, 1995, 114(2): 556-559.

[13] LU S Y, MA L B, WANG L C, et al. Hydrothermal synthesis and growth mechanism of basic magnesium chloride whiskers. Journal of Synthetic Crystals, 2016, 45(1): 170-176.

[14] LU B N, WANG S W, CHEN S P. Study on the property modification of compound magnesium hydroxide flame retardant. Applied Chemical Industry, 2012, 41(9): 1570-1574.

[15] LIU F M, XIA S P, ZHANG H Y. The study on the dehydration processes of bischofite and its complex salts during continuous heating by in-situ infrared spectroscopy. Spectroscopy and Spectral, 1988, 8(3): $8-14$.

[16] HUANG Z N, KOU S Z, JIN D D, et al. Surface adsorption and diffusion of Nb-Si-N nano-composite films: a first principles study. Journal of Functional Materials, 2015, 46(5): 5084-5088.

[17] DU G X, ZHENG S L, JIANG Q S, et al. Surface modification of ultrafine magnesium hydroxide powder. Industrial Minerals \& Processing, 2005, 34(9): 7-9.

[18] WANG B H, JING D C, WANG Z H, et al. Lattice distortion and abnormal infrared behavior of magnesium oxide nanorods. Henan Chemical Industry, 2008, 25(10): 25-27.

[19] KISSINGER H E. Reaction kinetics in differential thermal analysis. Analytical Chemistry, 1957, 29(11): 1702-1706. 